\title{
Capturing Dynamics in the Power Grid: Formulation of Dynamic State Estimation through Data Assimilation
}
N Zhou
S Elbert
Z Huang
S Wang
D Meng
R Diao

March 2014

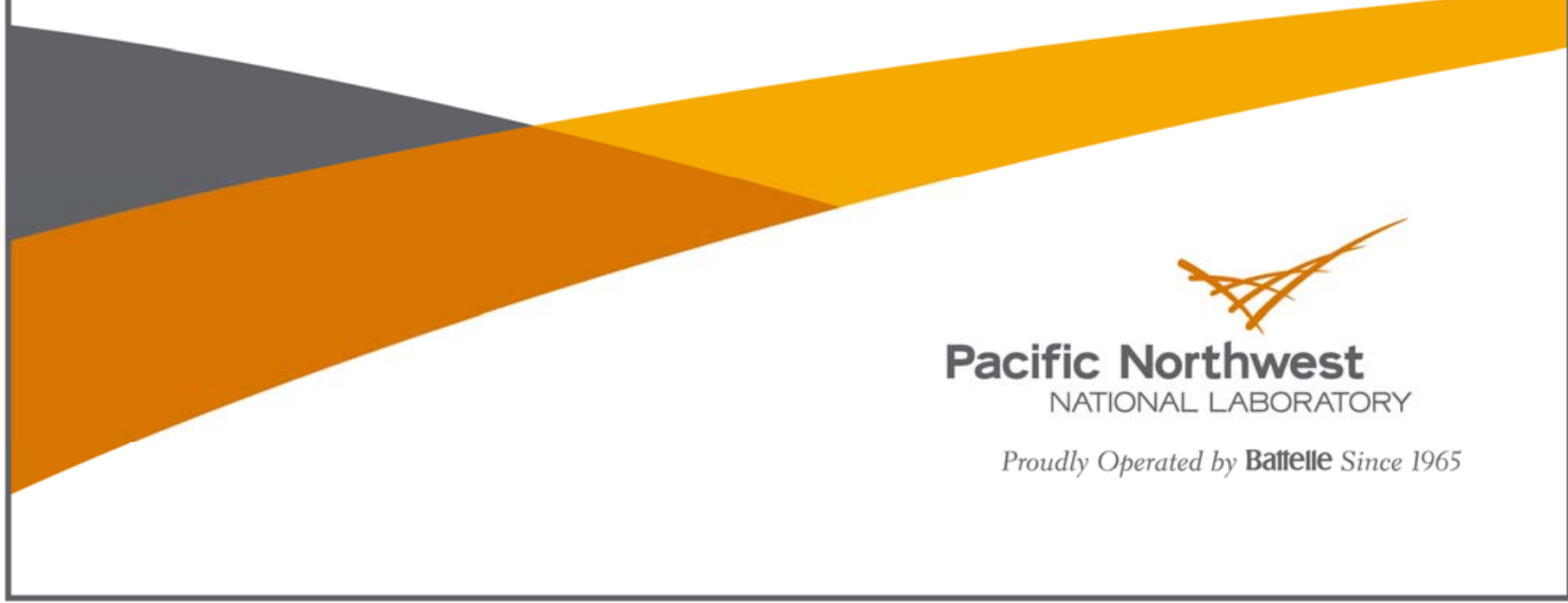




\title{
DISCLAIMER
}

This report was prepared as an account of work sponsored by an agency of the United States Government. Neither the United States Government nor any agency thereof, nor Battelle Memorial Institute, nor any of their employees, makes any warranty, express or implied, or assumes any legal liability or responsibility for the accuracy, completeness, or usefulness of any information, apparatus, product, or process disclosed, or represents that its use would not infringe privately owned rights. Reference herein to any specific commercial product, process, or service by trade name, trademark, manufacturer, or otherwise does not necessarily constitute or imply its endorsement, recommendation, or favoring by the United States Government or any agency thereof, or Battelle Memorial Institute. The views and opinions of authors expressed herein do not necessarily state or reflect those of the United States Government or any agency thereof.

\author{
PACIFIC NORTHWEST NATIONAL LABORATORY \\ operated by \\ BATTELLE \\ for the \\ UNITED STATES DEPARTMENT OF ENERGY \\ under Contract DE-AC05-76RL01830
}

Printed in the United States of America

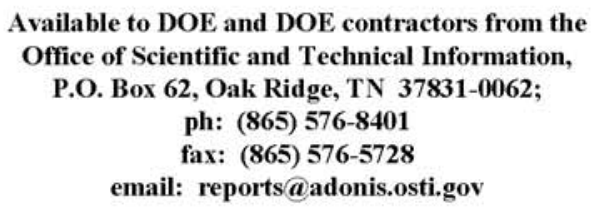

Available to the public from the National Technical Information Service 5301 Shawnee Rd., Alexandria, VA 22312 ph: (800) 553-NTIS (6847) email: orders $a$ ntis.gov $<$ http://www.ntis.gov/about/form.aspx > Online ordering: http://www.ntis.gov 


\section{Capturing Dynamics in the Power Grid: Formulation of Dynamic State Estimation through Data Assimilation}

$\begin{array}{ll}\text { N Zhou** } & \text { S Elbert* } \\ \text { Z Huang* } & \text { S Wang* } \\ \text { D Meng* } & \text { R Diao* }\end{array}$

March 2014

Prepared for

the U.S. Department of Energy

under Contract DE-AC05-76RL01830

*Pacific Northwest National Laboratory

Richland, Washington 99352

**Electrical and Computer Engineering Department,

Binghamton University, Binghamton, NY 13902 



\section{Executive Summary}

With the increasing complexity resulting from uncertainties and stochastic variations introduced by intermittent renewable energy sources, responsive loads, mobile consumption of plug-in vehicles, and new market designs, more and more dynamic behaviors are observed in everyday power system operation. To operate a power system efficiently and reliably, it is critical to adopt a dynamic paradigm so that effective control actions can be taken in time.

The dynamic paradigm needs to include three fundamental components: dynamic state estimation; look-ahead dynamic simulation; and dynamic contingency analysis (Figure 1.1). These three components answer three basic questions: where the system is; where the system is going; and how secure the system is against accidents. The dynamic state estimation provides a solid cornerstone to support the other 2 components and is the focus of this study.

Dynamic states (e.g., rotor angle and generator speed) are the minimum set of variables that can determine the status of a dynamic system. A dynamic model with accurate states can faithfully reveal system responses. Therefore, dynamic state estimation can provide a full dynamic view of a power grid and generate critical inputs for other operational tools.

To estimate the dynamic states of a power grid in real time, we developed and evaluated data assimilation methods to fuse phasor measurement unit (PMU) data with power system dynamic models. In this study, we defined a general dynamic state estimation problem for a power system and performance evaluation criteria. A problem is formulated for estimating the dynamic states of synchronous generators. As an initial effort, the following four data assimilation algorithms are developed, implemented and applied to estimate the dynamic states of a synchronous generator:

- Ensemble Kalman filter (EnKF)

- Extended Kalman filter (EKF)

- Unscented Kalman filter (UKF)

- Particle filter (PF)

By comparing their performance under statistical framework using Monte Carlo methods, it was found that

- The EnKF algorithm outperforms other algorithms when the typical PMU sampling rate is used for estimation.

- Measurement interpolation methods can improve the estimation accuracy of the EKF, UKF, and PF. The interpolation does not show significant influence on the performance of the EnKF.

- Increasing the number of samples can improve the estimation and convergence of the PF.

- All four algorithms are robust to missing data.

- The outliers cause some significant errors for all algorithms if the outliers are processed as normal data. The EKF, UKF, EnKF are more robust to the outliers than the PF. It takes more time for a PF to regain accurate state tracking after the outliers disappear. 
Based on these preliminary results, we will carry out the following studies to further enhance practicability by incorporating realistic conditions:

- Improve algorithm robustness against modeling and low data quality to improve estimation accuracy.

- Develop a realistic medium-size system for applying and testing the EnKF and other methods.

- Speed up computation and reduce computational requirement with acceptable estimation accuracy.

- Develop a framework for building a flexible, modular, extensible software suite that can be used in a range of environments.

The ultimate goal of these studies is to push forward for a real-world application. 


\section{Acronyms and Abbreviations}

$\begin{array}{ll}\text { EKF } & \text { extended Kalman filter } \\ \text { EnKF } & \text { ensemble Kalman filter } \\ \text { IEEE } & \text { Institute of Electrical and Electronics Engineers } \\ \text { MSE } & \text { mean squared error } \\ \text { PF } & \text { particle filter } \\ \text { PMU } & \text { phasor measurement unit } \\ \text { PST } & \text { Power System Toolbox } \\ \text { TVE } & \text { total vector error } \\ \text { UKF } & \text { unscented Kalman filter } \\ \text { WECC } & \text { Western Electricity Coordinating Council }\end{array}$





\section{Contents}

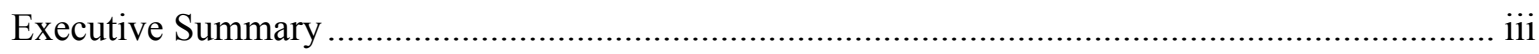

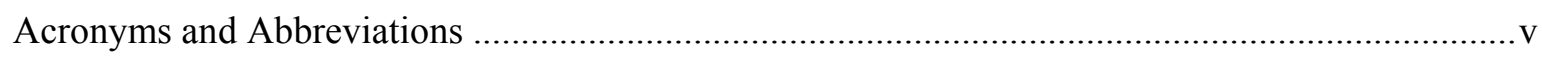

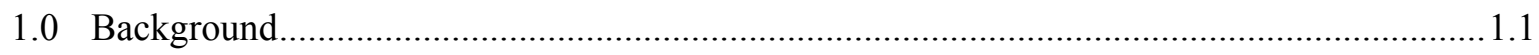

2.0 Introduction Based on Literature Review ..................................................................... 2.1

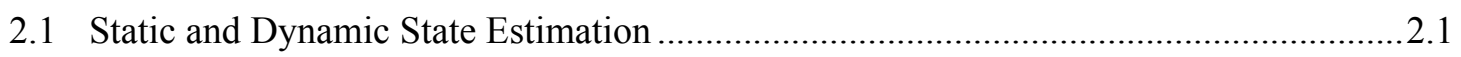

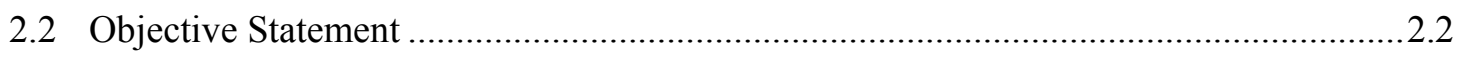

2.3 Current practice on dynamic state estimation …....................................................... 2.2

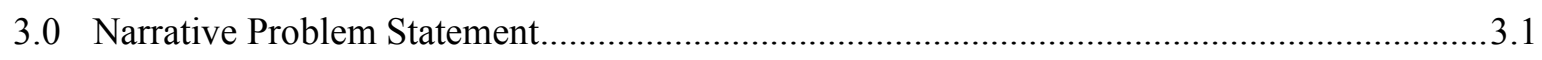

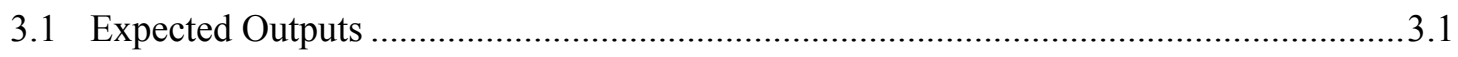

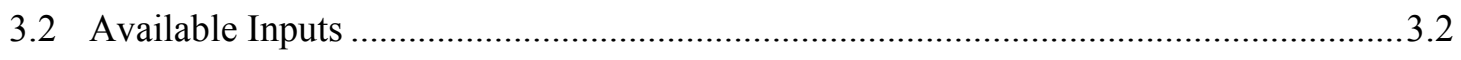

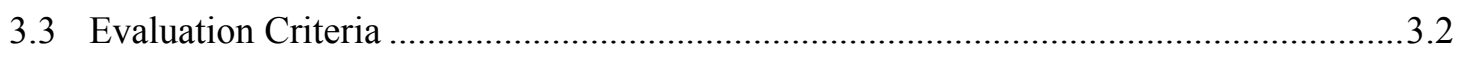

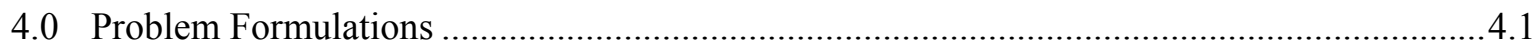

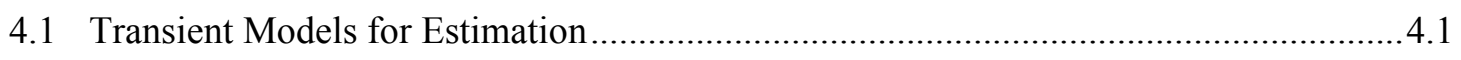

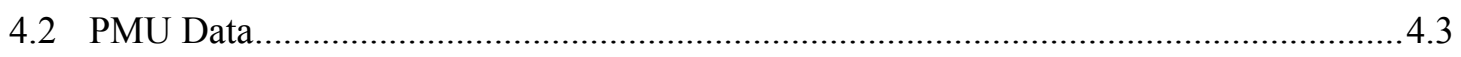

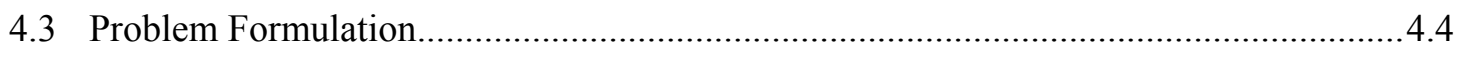

5.0 Solution Methods Using Data Assimilation Techniques ....................................................5.1

5.1 Overview of Data Assimilation in Other Domains ......................................................

5.2 Overview of Kalman Filter (UKF, EKF, EnKF, PF) .................................................

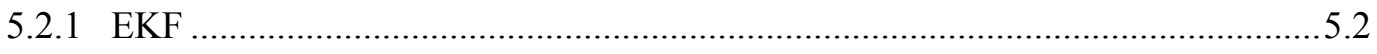

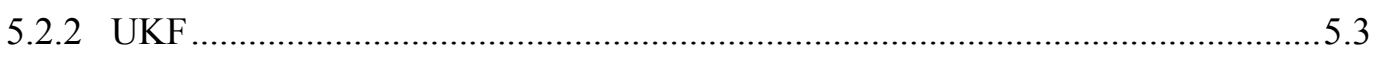

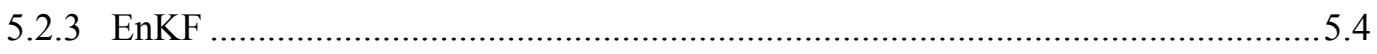

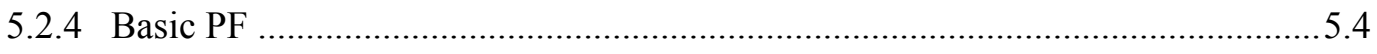

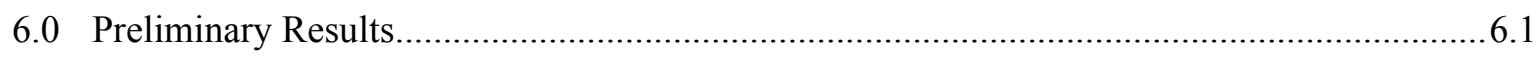

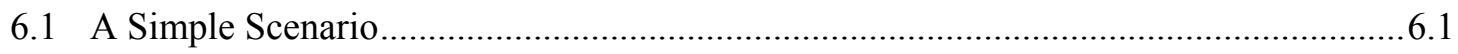

6.2 A Scenario with Measurement Interpolation............................................................ 6.3

6.3 A Realistic Scenario Considering Model Inadequacy ................................................ 6.4

6.4 A Realistic Scenario with Missing Data and Outliers .................................................... 6.5

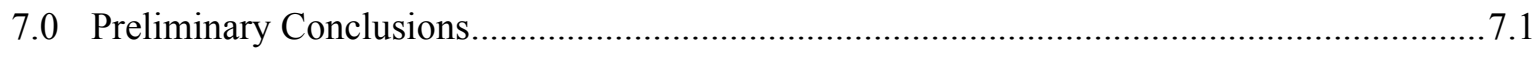

8.0 Scope of Further Study and Expected Outcome.................................................................. 8.1

8.1 Improve Algorithm Robustness against Modeling and Low Data Quality ..................... 8.1

8.2 Develop a Realistic Model for Applying and Testing EnKF using Field Measurement

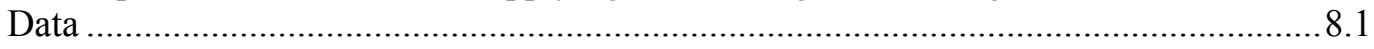

8.3 Speed Up Computation and Reduce Computational Requirement with Acceptable

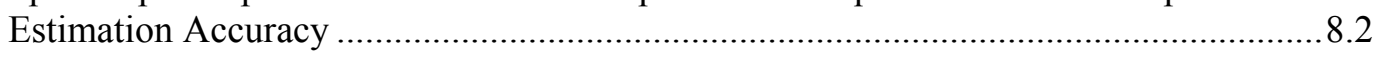

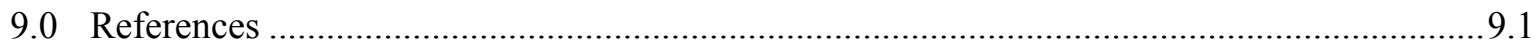




\section{Figures}

1.1 Integrated Dynamic Paradigm for Future Power Grid Operation Enabled by Dynamic State Estimation, Look-Ahead Dynamic Simulation and Real-Time Dynamic Contingency

Analysis

2.1 PMUs in the North American Power Grid............................................................................ 2.2

3.1 Problem Statement of Dynamic State Estimation................................................................ 3.1

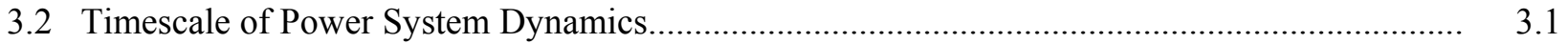

3.3 Illustration of Smoothing, Filtering and Prediction Methods ................................................... 3.2

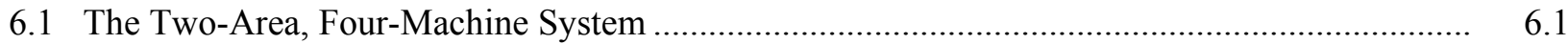

6.2 Comparison of MSEs from the EKF, EnKF, PF, and UKF for 100 Sets of Monte Carlo Simulations of a Simple Scenario

6.3 Estimated States from the EnKF with 200 Samples of 100 sets of Monte Carlo Simulations for a Simple Scenario.

6.4 Comparison of MSEs from the EKF, UKF, EnKF, and PF of 100 sets of Monte Carlo Simulations for the Scenarios with Measurement Interpolation and without Interpolation

6.5 Comparison of MSEs from the EKF, UKF, EnKF, and PF of 100 Sets of Monte Carlo Simulations for the Realistic Scenario

6.6 Comparison of MSEs from the EKF, UKF, EnKF, and PF of 100 Sets of Monte Carlo Simulations for the Realistic Scenario with and without Missing Data and Outliers

6.7 Estimated States from the EnKF of 100 Sets of Monte Carlo Simulations for the Realistic Scenario with Missing Data and Outliers 


\subsection{Background}

The electric power grid has been evolving over the last 120 years from a single power line to today's large networks. The evolution will continue at an accelerated rate with extensive smart grid development worldwide. In the next 10-15 years, a significant percentage of electricity will come from intermittent renewable sources, a large number of cars will be plugged into power grids, and a vast number of loads will actively respond to grid conditions and incentive signals. This development is largely driven by environmental and economic factors, such as reducing carbon emissions and saving electricity cost for consumers. The result is new stochastic behaviors and dynamics that the grid has never seen nor been designed for. Operating such a dynamic grid with sufficient reliability and efficiency is a monumental challenge.

Traditionally, a quasi-steady-state assumption is applied to operation studies and decision making to simplify operation models and reduce computational complexity. Today's operation is primarily based on a model that largely ignores dynamics in the power grid. Electromechanical interaction of generators and dynamic characteristics of loads and control devices are not included in operational models. The quasisteady-state assumption reduces the computation complexity by several orders of magnitude and makes operation studies on serial computers feasible within the required operational time intervals. This assumption was reasonable and historically has contributed significantly to the development of power grid computational methods and tools. However, with the rapid evolution of grid requirements and computing technologies, it is important to reexamine this assumption for improving grid operation.

Because of the quasi-steady-state assumption, many dynamic studies cannot be performed in an operational environment. Smart grid development makes the grid much less quasi-steady-state and more dynamic, compared to the traditional power grid. For future power grids it is essential to establish a dynamic operation paradigm relative to today's steady-state model.

The dynamic paradigm needs to include three fundamental components: dynamic state estimation, look-ahead dynamic simulation, and dynamic contingency analysis (Figure 1.1). These three components answer three basic questions: where the system is, where the system is going, and how secure the system is against accidents. The dynamic state estimation provides a solid cornerstone to support the other two components and is the focus of this study.

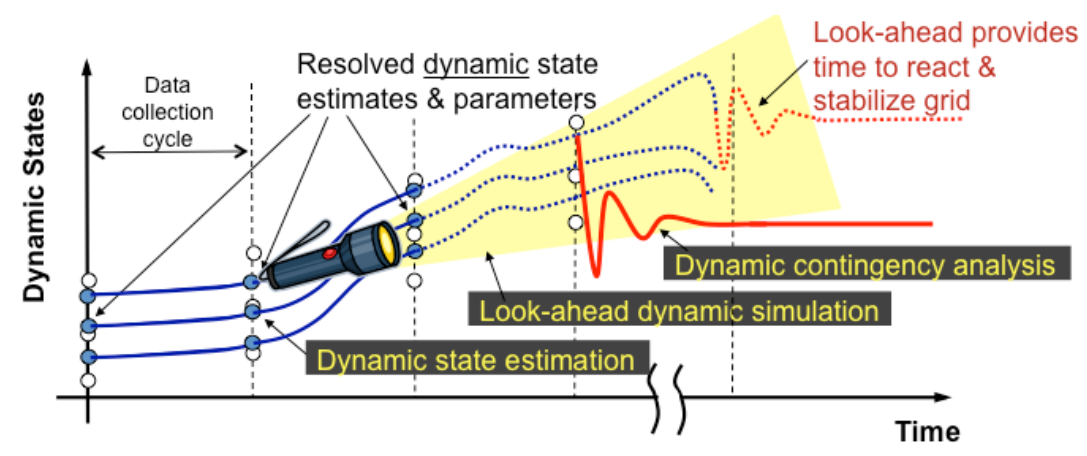

Figure 1.1. Integrated Dynamic Paradigm for Future Power Grid Operation Enabled by Dynamic State Estimation, Look-Ahead Dynamic Simulation and Real-Time Dynamic Contingency Analysis 



\subsection{Introduction Based on Literature Review}

State estimation - a central function in power grid operations - generates critical inputs for other operational tools, such as contingency analysis, automatic generation control, and optimal power flow (Arbur 2004, Monticelli 2000). Any inaccuracy or deficiency introduced in the state estimation process will be propagated and possibly exaggerated through these tools and greatly affect system operation efficiency.

\subsection{Static and Dynamic State Estimation}

Traditional state estimators receive telemetered data from a supervisory control and data acquisition (SCADA) system, which are sampled in the time interval of several seconds. The SCADA data are used with a steady-state power flow model to estimate a set of static state variables, i.e., bus voltages and phase angles. Because the power flow model ignores the dynamic transitions, only the static states of the power system are estimated, i.e., only a series of snapshots of the system conditions are generated and the dynamic transition between the snapshots is not considered.

Dynamic states (e.g., rotor angle and generator speed) are the minimum set of variables that can determine the status of a dynamic system. A dynamic model with accurate states can faithfully reveal system responses. Therefore, Dynamic state estimation provides a full dynamic view of a power grid, which further enables real-time dynamic simulation and dynamic contingency analysis and wide-area control.

Dynamic state estimation is made possible by the availability of high quality phasor measurement unit (PMU) data and increasing computational capabilities. PMU data has a typical sampling rate of 30 or 60 samples per second, is well synchronized with the Global Positioning System clock, and can continuously capture the dynamic response of a power system under normal and abnormal conditions; thus it can enable dynamic state estimation. In the North American grid, almost 1700 PMUs had been deployed by 2013 (Silverstein 2013) (Figure 2.1). The PMU data provides a solid cornerstone for dynamic state estimation. Computing hardware and software technologies have been significantly advanced in the last decade. Computational throughput is being driven by the large-scale use of multicore processors, with hundreds of thousands of cores in a large-scale high performance computer. The challenge is how to assimilate PMU data into a dynamic model for estimating dynamic states and how to utilize advanced computing technologies to perform analysis in real time. In our work, we use the parallel filter method to address these challenges. 


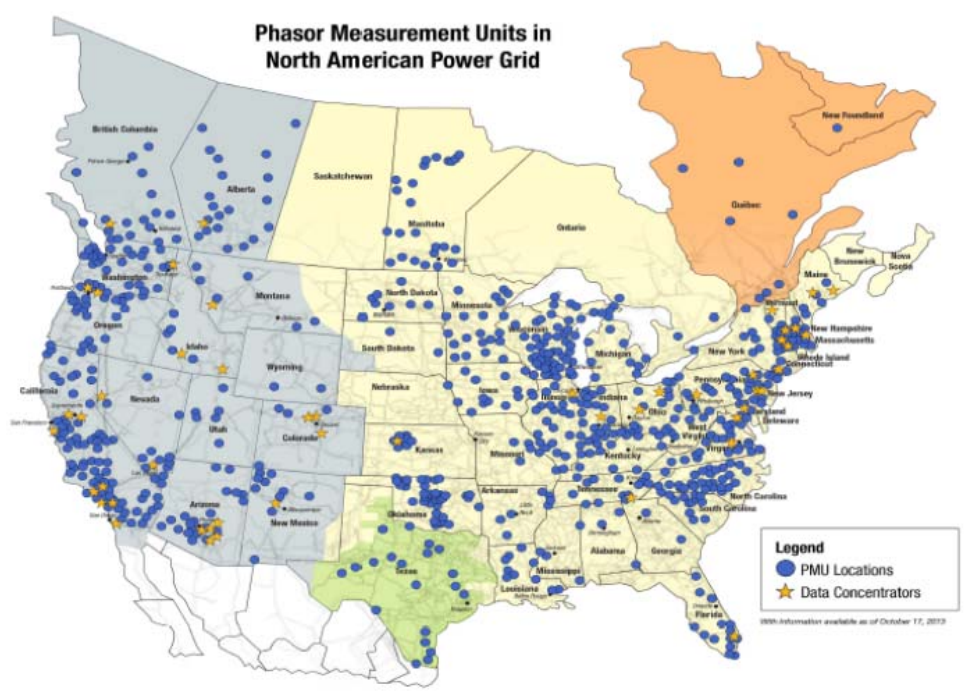

Figure 2.1. PMUs in the North American Power Grid (Silverstein 2013)

\subsection{Objective Statement}

The objective of this study is to develop fundamental technologies of dynamic state estimation for power grid operations. Methods to estimate the real-time dynamic states of the power grid will be developed, implemented in a high performance computing environment and evaluated in a practical power system. The performance of the developed methods will be evaluated in terms of estimation accuracy and computing efficiency for helping real-time operational decisions.

\subsection{Current Practice on Dynamic State Estimation}

Some initial studies have been carried out to explore the feasibility of the dynamic state estimation. Huang et al. (2009, 2013) and Fan and Wehbe (2013) used an extended Kalman filter (EKF) for online dynamic state estimation. Ghahremani and Kamwa (2011a) used an EKF to simultaneously estimate the generator states and unknown inputs. Ghahremani and Kamwa (2011b) used an unscented Kalman filter (UKF) to estimate the dynamic states of a single-machine infinite bus system. Zhou et al. (2012) proposed an ensemble Kalman filter (EnKF) method to simultaneously estate the states and parameters. Zhou et al. (2013) proposed an extended particle filter (PF) to estimate the dynamic states.

These studies demonstrated the value and feasibility of estimating dynamic states using PMU data with Bayesian-based filtering approaches. However, how to address model size, model errors, limited data availability, and statistical performance issues for a real-world application is still a big challenge. First, robust estimation methods that perform well over a wide range of noise scenarios are needed. Existing works usually focus on performance of a simple case, for example, single noise instance has been used to evaluate performance (Huang et at. [2007], Huang et al. [2009], Fan and Wehbe [2013] and Ghahremani and Kamwa [2011a,b]). Second, to implement effective controls in real time for a real-world system, states need to be estimated for a realistic-size system in real time, while the current studies focus on small problems with two machines, four machines, or 16 machines using serial computers. For the real-world application, the performance scalability of data assimilation methods needs to be evaluated 
with a medium-size realistic system with modern parallel computation capability. Third, the above studies assume that PMU data are available at any desired locations, while in practice the PMUs have been deployed and the location cannot be readily changed. Therefore, before estimating a state, a data assimilation method needs to be evaluated under a practical PMU setup. Fourth, in a real-world application, a dynamic model is only a simplified description of a real system. A practical dynamic state estimation method must tolerate the model noise to be applied reliably. Yet, in most studies, the models for estimating states have similar structure to the models for generating simulation data. This practice does not give enough consideration to how model noises may influence the estimation. With these technical gaps in mind, we define a dynamic state estimation problem for a power system. 



\subsection{Narrative Problem Statement}

In this section, we define a dynamic state estimation problem for a power system. In the problem statement, we clarify the expected outcome of dynamic state estimation, available input, and evaluation criteria. The structure of the problem statement is illustrated by Figure 3.1. The problem statement is used to develop and evaluate potential solutions.

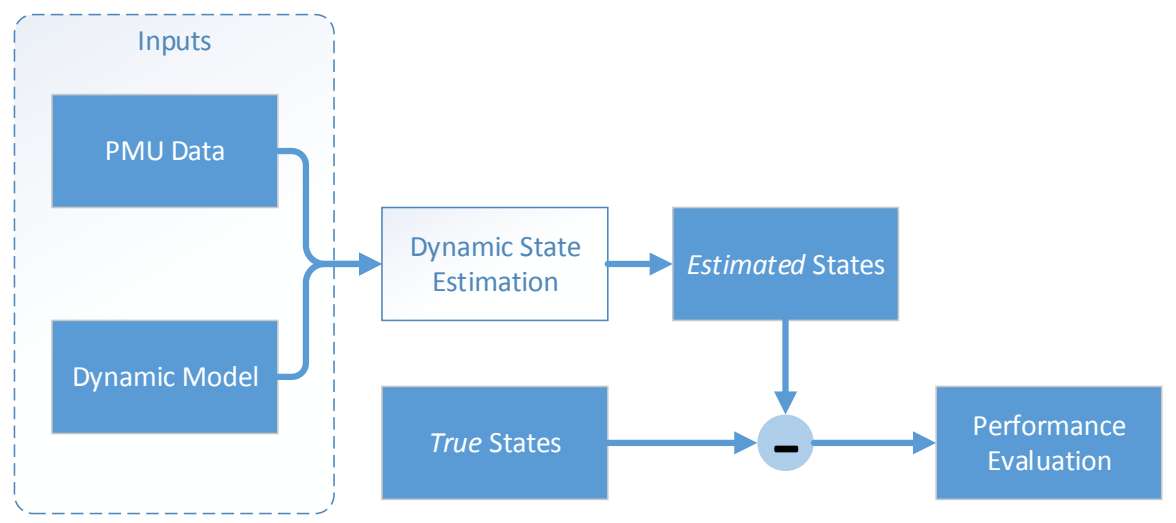

Figure 3.1. Problem Statement of Dynamic State Estimation

\subsection{Expected Outputs}

The goal of dynamic state estimation is to estimate the electromechanical dynamic states of a power system. Dynamic states are the minimum set of variables that can determine the status of a dynamic system (DeRusso et al. 1998) for a time scale of interest. For the electromechanical dynamics which we are interested in, the time scale is at a level between 100 milliseconds and 1 second (Figure 3.2). The electromechanical dynamic states can be used to enhance the rotor angle stability of a power system by enabling dynamic contingency analysis and state prediction. Note that states are different from parameters in that parameters often remain a constant value while states may keep changing.

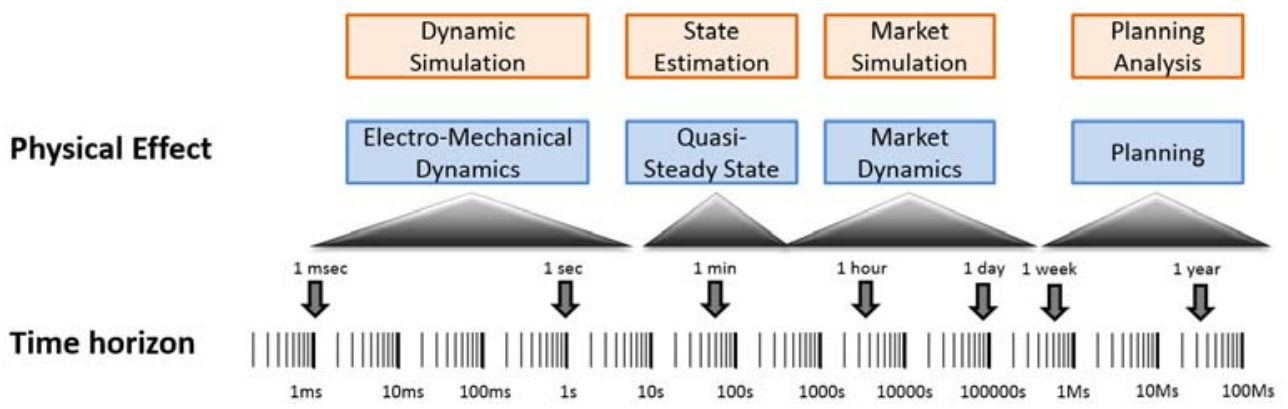

Figure 3.2. Timescale of Power System Dynamics

Depending on the requirement of an application, the states can be estimated for past, current and future time (Figure 3.3). A smoothing method estimates the states of past time and can be used to reproduce past events for forensic studies. A filtering method estimates the states at the current time and can be used to drive control signals to improve system stability. A prediction method estimates the states 
of future time and can be used to guide proactive remedial actions. The ultimate goal of this study is to enhance power system stability in real time, and therefore the focus is placed on the filtering method.

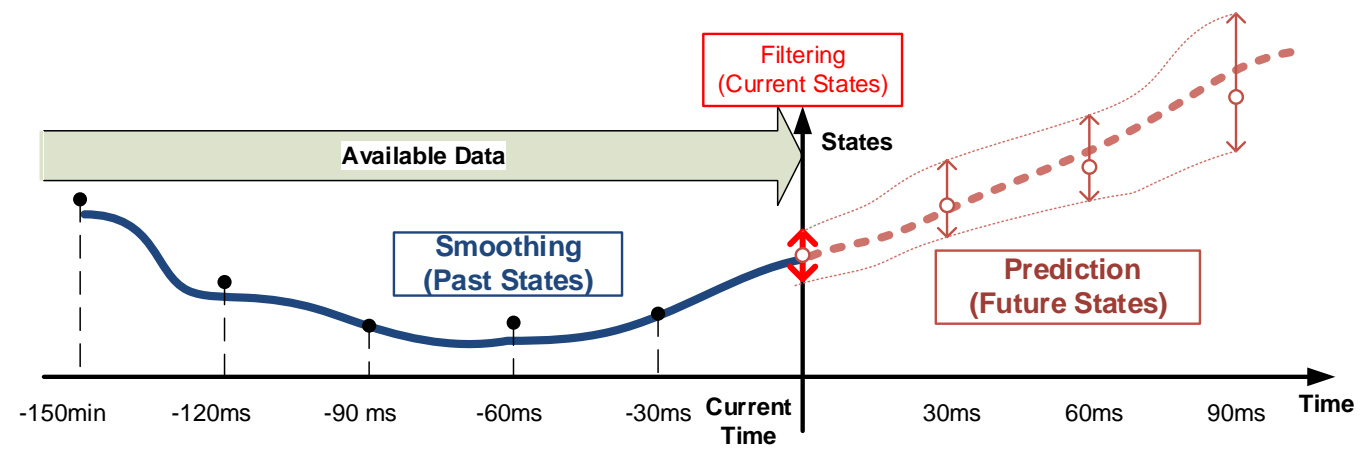

Figure 3.3. Illustration of Smoothing, Filtering and Prediction Methods

\subsection{Available Inputs}

To estimate the current dynamic states, only the data and models that are available up to the current time can be used as the inputs of a filtering method. All the PMU data available up to the current time can be used. Dynamic models that are available may also be used. Studies shall be carried out with proper considerations of the practical constraints of models and data.

1. PMU data:

a. PMU data have measurement noise. The measurement noises are defined as total vector errors (TVEs). For a PMU that follows Institute of Electrical and Electronics Engineers (IEEE) Standard $\mathrm{C} 37.118$, the device error shall be smaller than $1 \%$, in addition to the noise incurred by the PMU device (Martin et al. 2008). Additional noise is often introduced by current transformers (CT) and potential transformers (PT).

b. Because of the cost of PMU installation, there are limited numbers of PMUs available. Compared to the size of a power grid, PMU data are sparse and mainly available on high voltage lines.

c. Because of unexpected sensor and data communication failures, outliers and missing data are not uncommon in PMU data.

2. Dynamic models

a. Dynamic models are often available to describe the dynamic features of a power system. The dynamic models are only an approximate description of a power system. Therefore, the system responses and model responses to a stimulation are often different at various levels. The response difference reveals the modeling noises and deficiencies, and must be considered for a real application.

\subsection{Evaluation Criteria}

There are many algorithms for estimating dynamic states of a power system. Evaluation criteria are needed to evaluate and compare the estimation performance and select a proper estimation algorithm. The following measurements can be applied to quantify the performance of a dynamic state estimation algorithm under a statistical framework. 
1. Accuracy: Accuracy is defined as the difference between estimated states and true states. However, because the true states are unknown in a real power system, several indexes based on measurement residuals will be used to evaluate state estimation accuracy (Guo et al. 2013, Al-Othman and Irving 2006).

2. Robustness against modeling errors and measurement errors: The robustness of an algorithm can be measured by its tolerance of noise in a model and data. In practice, the robustness of an algorithm is evaluated by the maximum modeling errors or measurement errors under which the algorithm can still achieve the required state estimation accuracy.

3. Speed of computation: The speed of an algorithm can be measured by the capability of an algorithm to keep up with the speed of inflowing data. Ideally, the results are available before the next set of data arrives. This is not currently possible for large real-world systems. In this case it may be necessary to use multiple computing systems to maintain a constant latency of results in order to process all the data.

4. Scalability: Scalability of an algorithm refers its effectiveness when it is applied a larger system. There are multiple aspects that need to be considered, including serial scalability, parallel scalability, and accuracy. Serial scalability determines how much longer a calculation will take when the problem size is increased but the amount of computation is not. For example, if the problem size doubles but the solution takes four times as long, the algorithm is said to scale as the square of the problem size. Algorithms that scale linearly or less, e.g., logarithmically, are highly desirable. Parallel scalability is often considered in terms of strong and weak scaling. In strong scaling, the time required to solve a problem is directly proportional to the amount of computation being used, i.e., two processors can solve the problem twice as fast as one processor, ten processors ten times as fast, etc. Amdahl's law, which states that parallel speedup is limited by the amount of serial computing required by an algorithm, makes the range of strong scaling generally very limited. Weak scaling, on the other hand, allows the use of more processors on bigger problems because the serial sections scale differently than the parallel sections. Eventually, however, adding more processors actually slows the rate at which results are generated. Algorithms that slow down later rather than sooner are obviously preferred. An algorithm that performs fewer arithmetic operations (multiplies and adds) may not scale as well as the one that does more operations if it has to move more data between processors, so the scalability of an algorithm's compute-to-communicate ratio is important. Finally, a robust, numerically stable algorithm may be able to solve larger problems than one that is computationally more efficient but numerically unstable, e.g., one that takes the differences of small numbers - a process that can destroy numerical precision. Scalability at all levels is critical because methods that work for small problems may fall apart when applied to large real-world problems. 



\subsection{Problem Formulations}

In this section, we formulate a specific dynamic state estimation problem to estimate dynamic states of a synchronous generator. We will discuss the dynamic models of a generator, dynamic states to be estimated, available PMU data, and objective functions.

\subsection{Transient Models for Estimation}

This subsection introduces estimation models used by filtering algorithms for estimating dynamic states of a synchronous machine. Also, to apply a filtering method for discrete measurements, a modified Euler method is applied to discretize the continuous model (Kundur 1994).

1. Continuous-time models for generators

A fourth-order model (1) can be used to model a synchronous machine in local $d$ - $q$ reference frame to estimate the states (Kundur 1994).

$$
\left\{\begin{array}{l}
\dot{\delta}=\omega_{0} \Delta \omega \\
\Delta \dot{\omega}=\frac{1}{2 H}\left(T_{m}-T_{e}-K_{D} \Delta \omega\right) \\
\dot{e}_{q}^{\prime}=\frac{1}{T_{d 0}^{\prime}}\left(E_{f d}-e_{q}^{\prime}-\left(x_{d}-x_{d}^{\prime}\right) i_{d}\right) \\
\dot{e}_{d}^{\prime}=\frac{1}{T_{q 0}^{\prime}}\left(-e_{d}^{\prime}+\left(x_{q}-x_{q}^{\prime}\right) i_{q}\right)
\end{array}\right.
$$

In (1), $\delta$ is the rotor angles in radians, by which the local $q$ axis leads the global $R$ axis (Kundur 1994); $\Delta \omega$ is rotor speed deviation; and $e_{d}{ }^{\prime}$ and $e_{q}{ }^{\prime}$ are the transient voltages along $q$ and $d$ axes. The parameter $T_{m}$ is the mechanical torque; $T_{e}$ is the electric air-gap torque; and $E_{f d}$ is the internal field voltage. The parameter $\omega_{0}$ is the rated value of the angular frequency; $H$ is the inertia constant, and $K_{D}$ is the damping factor. The parameters $T_{d 0}{ }^{\prime}$ and $T_{q 0}{ }^{\prime}$ are the open circuit time constants in the directions of the $d$ and $q$ axes respectively; $x_{d}$ and $x_{q}$ are the synchronous reactance at the $d$ and $q$ axes respectively; and $x_{d}{ }^{\prime}$ and $x_{q}{ }^{\prime}$ are the transient reactance at the $d$ and $q$ axes respectively.

To facilitate the notation, (1) is transformed into a general state space model as given in (2) and (3).

$$
\begin{aligned}
& \left\{\begin{array}{c}
\dot{x}=f_{c}(x, u)+w_{c} \\
y=h_{c}(x, u)+v_{c}
\end{array}\right. \\
& E\left[w_{c} w_{c}^{T}\right]=Q \\
& E\left[v_{c} v_{c}^{T}\right]=R
\end{aligned}
$$




$$
\begin{aligned}
& x=\left[\begin{array}{llll}
\delta & \Delta \omega & e_{q}^{\prime} & e_{d}^{\prime}
\end{array}\right]^{T} \\
& u=\left[\begin{array}{llll}
T_{m} & E_{f d} & i_{R} & i_{I}
\end{array}\right]^{T} \\
& y=\left[\begin{array}{lll}
e_{R} & e_{I}
\end{array}\right]^{T}
\end{aligned}
$$

In (2)-(3) $x$ is the state vector, $u$ is the input vector, and $y$ is the output vector. Functions $f_{c}$ (*) and $\left.h_{c}{ }^{*}\right)$ are the state transition and output functions, respectively. The subscript " $c$ " indicates the continuous-time model. The vectors $w_{c}$ and ${ }_{v_{c}}$ represent the process and output noise, respectively. They are modeled as Gaussian white noise whose covariance matrices are defined by (2.c) and (2.d) as Q and R. $E[*]$ represents statistical expectation. To transform (1) into $f_{c}(*)$ in (2.a), $i_{d}, i_{q}$, and $T_{e}$ were written as functions of $x$ and $u$ using (4).

$$
\begin{aligned}
& i_{d}=i_{R} \sin \delta-i_{I} \cos \delta \\
& i_{q}=i_{I} \sin \delta+i_{R} \cos \delta \\
& T_{e} \approx P_{t}=\left(e_{d}^{\prime}+i_{q} x_{q}^{\prime}\right) i_{d}+\left(e_{q}^{\prime}-i_{d} x_{d}^{\prime}\right) i_{q}
\end{aligned}
$$

Similarly, to implement output function $h_{c}(*)$ in (2.b), $e_{I}$ and $e_{R}$ were written as functions of $x$ and $u$ using (5). Note that $i_{d}$ and $i_{q}$ in (5) are the functions of $i_{R}$ and $i_{I}$ as in (4.a) and (4.b). The model defined by (1), (4), and (5) is then discretized and used for estimating states.

$$
\begin{aligned}
& e_{R}=\left(e_{d}^{\prime}+i_{q} x_{q}^{\prime}\right) \sin \delta+\left(e_{q}^{\prime}-i_{d} x_{d}^{\prime}\right) \cos \delta \\
& e_{I}=\left(e_{q}^{\prime}-i_{d} x_{d}^{\prime}\right) \sin \delta-\left(e_{d}^{\prime}+i_{q} x_{q}^{\prime}\right) \cos \delta
\end{aligned}
$$

\section{Discretization method and discrete time models}

To estimate states using the discrete measurements, the continuous-time model in (2) was discretized into a discrete time model (6), where the subscript $k$ indicates the time at $k \Delta t$.

$$
\left\{\begin{array}{l}
x_{k}=f\left(x_{k-1}, u_{k-1}\right)+w_{k-1} \\
y_{k}=h\left(x_{k}, u_{k}\right)+v_{k}
\end{array}\right.
$$

More specifically, the state transition function (2.a) was discretized by applying the modified Euler method (Zhou et al. 2013) using (7). In (7), $\widetilde{f}_{c}$ can be calculated by (8). When $\Delta t$ is small enough, the discrete process noise $w_{k-1}$ can be approximated by (9). Because the continuous-time process noise $w_{c}$ is defined by (2.c), the mean of $w_{k-1}$ is 0 and the covariance of $w_{k-1}$ can be calculated as (10). Equation (10) indicates that the variance of process noise can be increased proportionally with the sampling interval $\Delta t$ during the state estimation.

$$
x_{k} \approx x_{k-1}+\widetilde{f}_{c} \Delta t+w_{k-1}
$$




$$
\begin{aligned}
& \left\{\begin{array}{l}
\tilde{f}_{c}=\left(f_{c}\left(\tilde{x}_{k}, u_{k}\right)+f_{c}\left(x_{k-1}, u_{k}\right)\right) / 2 \\
\tilde{x}_{k}=x_{k-1}+\Delta t \cdot f_{c}\left(x_{k-1}, u_{k-1}\right)
\end{array}\right. \\
& w_{k-1} \approx \int_{(k-1) \Delta t}^{k \Delta t} w_{c}(\tau) d \tau \\
& Q_{d} \stackrel{\Delta}{=} E\left(w_{k-1} w_{k-1}^{T}\right) \\
& =\int_{(k-1) \Delta t}^{k \Delta t} \int_{(k-1) \Delta t}^{k \Delta t} E\left[w_{c}\left(\tau_{1}\right) w_{c}^{T}\left(\tau_{2}\right)\right] d \tau_{1} d \tau_{2} \\
& =\int_{(k-1) \Delta t}^{k \Delta t} \int_{(k-1) \Delta t}^{k \Delta t} Q \delta\left(\tau_{1}-\tau_{2}\right) d \tau_{1} d \tau_{2}=Q \Delta t
\end{aligned}
$$

Measurement equation (2.b) can be discretized into (11). Here, $v_{k}$ is the discrete time output noise. Because the continuous-time output noise $v_{c}$ is defined by (2.b), the mean value of $v_{k}$ is 0 . The covariance of $v_{k}$ depends on how measurement instruments are set up. To simplify the study, this report assumes no prefilter. Therefore, the covariance of the $v_{k}$ may be computed using (12) (Schinkel et al. 2003).

$$
\begin{aligned}
& y_{k}=h_{c}\left(x_{k}, u_{k}\right)+v_{k} \\
& R_{d} \stackrel{\Delta}{=} E\left(v_{k} v_{k}^{T}\right)=R
\end{aligned}
$$

\subsection{PMU Data}

PMUs are deployed in power systems to measure the voltage and the current phasors at major transmission lines. PMU data have a typical sampling rate of 30 or 60 samples per second, are well synchronized with the Global Positioning System clock, and can continuously capture the dynamic response of a power system under normal and abnormal conditions. According to IEEE Standard C37.118-2005 (Martin et al. 2008), TVEs are used to quantify the accuracy of a PMU. Measurement noise may also include the noise from current transformers and potential transformers.

In addition to normal measurement noises, it is not uncommon for PMU measurements to be corrupted by missing data and outliers. Missing data may be caused by temporary communication failures, and is often identified and marked out by error detection schemes associated with communication protocols. Outliers are measurements with significantly large measurement errors that may be caused by extraordinary disturbances or temporary sensor failures. By checking the residuals, an outlier detector may be able to detect some outliers. Yet, because such an outlier detector needs to maintain a balance between correct and erroneous determinations (false positives and false negatives), it

will not be able to detect all outliers. Undetectable outliers (false negatives) carry misleading information and present a major challenge to state estimation algorithms.

Typically there are two categories of data that are often used in estimating dynamic states of a power system: simulation data and field measurement data 


\section{Simulation data:}

Simulation data are generated by performing dynamic simulation using a dynamic power system model. Note that the simulation model may be different from the estimation model discussed in the previous subsection. A simulation model is for mimicking system behaviors while an estimation model is for estimating states. There are several advantages of using simulation data. i) For a simulation data set, the true states are available. Therefore, it is easy to evaluate the accuracy of an estimation. ii) It is easy to set up a large number of representative scenarios to study the statistical performance of an estimation method. Often, Monte Carlo methods are used to evaluate the average performance. iii) It is easy to test the applicability of an estimation method on different systems because switching among different systems is easy for simulation studies. iv) Measurements can be generated at any desired points for simulation data. Therefore, the influence of certain measurements on the estimation results can be readily evaluated. Because of these advantages, simulation data have been used in almost all the studies to evaluate algorithm performance.

One caveat is that the quality of simulation data depends on how well a model describes a system. The responses from a simulation model are different from a system's responses to some degree. Therefore, field measurement data shall be used to test the applicability of a state estimation method in a real-world application.

\section{Field measurement data}

Field measurement data are collected from sensors deployed into a system. The measurement data carry invaluable information about the status of a real system. Information and knowledge extracted from field measurement data can improve situational awareness of a power system operator and directly support real-time decision making in power system operations.

Unlike simulation data, there are many more uncertainties in field measurement data. For example, is the noise in field measurement Gaussian or non-Gaussian? How do these uncertainties impact dynamic estimation methods? Field measurement data requires that state estimation methods be highly robust against noise. Ultimately, a dynamic state estimation method needs to be tested using real-world data to evaluate its applicability.

\subsection{Problem Formulation}

We formulate a real-time dynamic state estimation problem for synchronous generators as follows. Assuming a sampling interval of $\Delta t$ seconds, given measurements of voltage phasors, current phasors at some locations for time instance of $t=\Delta t, 2 \Delta t, 3 \Delta t \cdots, k \Delta t$, estimate the synchronous machine's states $\delta(k \Delta t), \Delta \omega(k \Delta t), e_{q}^{\prime}(k \Delta t)$ and $e_{d}^{\prime}(k \Delta t)$. A user may choose a dynamic model for the estimation. 


\subsection{Solution Methods Using Data Assimilation Techniques}

The Kalman filter is the most widely used Bayesian-based data assimilation method. It was named after Rudolf Kalman, who published his famous recursive method to estimate dynamic states of a linear system (Kalman 1960). Assuming Gaussian noise, the Kalman filter provides minimum-variance estimates of states through a recursive approach.

In addition to its original successful applications in linear systems, there are many publications extending the Kalman filter to nonlinear systems. The major difference among different nonlinear Kalman-filter methods is their approach to propagating the mean and covariance of the dynamic states. The EKF (Welch and Bishop 2006, Shih and Huang 2002) linearizes the state space model using a firstorder approximation. The mean and covariance of states are propagated using Jacobian matrices. The UKF (Wan and van der Merwe 2001) propagates the mean and covariance of states using a deterministicsampling approach to achieve a second-order approximation. The EnKF propagates the mean and covariance of states using a Monte Carlo sampling approach (Evensen 1994). In the EnKF, the distribution of the states is represented by a collection of samples, referred to as ensembles. All the above Kalman filters assume the joint Gaussian distribution of both measurements and states, and use the Bayesian approach to derive the Kalman gain. In contrast, the PF (Arulampalam et al. 2002) is a more general Bayesian approach, which does not rely on Gaussian noise assumption. Similar to the EnKF, the $\mathrm{PF}$ also uses the samples (also known as particles) to represent the probability distribution of random variables. Different from the EnKF, the PF directly corrects the states without assuming Gaussian distribution. This general approach is more applicable to highly nonlinear systems. However, the PF usually requires a very large number of samples and therefore is difficult to apply to high-dimensional systems.

\subsection{Overview of Data Assimilation in Other Domains}

The goal of data assimilation is to fuse model and data together to get better results than using model or data alone. Data assimilation has been widely studied in many scientific fields, especially in the fields of geosciences, hydrology and weather forecasting. For example, data assimilation has been used to integrate atmospheric models with radiosonde and satellite observations (Houtekamer and Mitchel11998). It is also used in oceanography to integrate ocean model with radar data. (Hoteit and Pham 2004).

Models used in data assimilation can be classified as deterministic or stochastic according to whether randomness is considered in the system. They may also be classified as static or dynamic models depending on whether continuous time-dependent change is accounted for. Different methods have been developed for data assimilation for different types of models. In the least squares method and its variants, for example, generalized least squares are commonly used to deal with deterministic data assimilation problems. As for statistical estimation, Fisher's maximum likelihood techniques and the Bayesian approach, including maximum a posteriori (MAP) estimate and minimum-variance estimate, form two basic frameworks of assimilation methods. Under the Bayesian framework, the Kalman filter, a recursive version of a minimum-variance estimator, is becoming a standard method for data assimilation (Houtekamer and Mitchell 1998). 


\subsection{Overview of Kalman Filter (UKF, EKF, EnKF, PF)}

Under a Bayesian framework, the implementations of theses algorithms have a similar structure. After initialization, all the filtering algorithms assimilate one snapshot of data at every time step. For one snapshot of data, there are two steps: a prediction step and a correction step. In the prediction step, the mean and covariance of states at time step $k$ are predicted based on the states at step $k-1$. In the correction step, the predicted mean and covariance are corrected based on new measurements obtained at time step $k$. The algorithms for implementing these filtering methods are detailed as follows.

\subsubsection{EKF}

The EKF linearizes the system at the current operating point using the Jacobian matrices as in (13), (14) and (15) (Welch and Bishop 2006).

\section{EKF Prediction:}

$$
\begin{aligned}
& x_{k}^{-}=f\left(x_{k-1}, u_{k-1}\right) \quad k \geq 1 \\
& P_{k}^{-}=F_{k-1} P_{k-1} F_{k-1}^{T}+Q_{d}
\end{aligned}
$$

\section{EKF Correction:}

$$
\begin{aligned}
& x_{k}=x_{k}^{-}+K_{k} \tilde{y}_{k} \\
& K_{k}=P_{k}^{-} H_{k}^{T}\left(H_{k} P_{k}^{-} H_{k}^{T}+R_{d}\right)^{-1} \\
& \tilde{y}_{k}=z_{k}-h\left(x_{k}^{-}, u_{k}^{-}\right) \quad k \geq 1 \\
& P_{k}=\left(I-K_{k} H_{k}\right) P_{k}^{-}
\end{aligned}
$$

where $x_{k}^{-}$and $P_{k}^{-}$are known as the a priori mean and covariance (of the states) respectively. They are estimated from the data up to time step $k-1$. The symbols $x_{k}$ and $P_{k}$ are known as the a posteriori mean and covariance of the states, respectively, which are derived by adding the information from $z_{k}$ to $x_{k}^{-}$and $P_{k}^{-}$. The symbol $K_{k}$ is the Kalman gain. The symbol $\tilde{y}_{k}$ is the residual between estimate $h\left(x_{k-1}^{-}, V_{k-1}\right)$ and measurement $z_{k}$. $F_{k}$ and $H_{k}$ are Jacobian matrices defined by (15). A perturbation approach is used to numerally derive the Jacobian matrices in this report.

$$
\begin{aligned}
& F_{k-1}=\left.\frac{\partial f}{\partial x}\right|_{X_{k}^{-}} \\
& H_{k}=\left.\frac{\partial h}{\partial x}\right|_{x_{k}^{-}}
\end{aligned}
$$




\subsubsection{UKF}

The UKF uses an unscented transform to pick a set of samples to represent the probability distribution of states and propagates these samples through the nonlinear functions $f$ and $h$ to reconstruct the mean and covariance. The UKF estimation method is summarized as (16) and (17) (Wan and van der Merwe 2001).

\section{UKF Prediction}

$$
\begin{aligned}
& x_{k}^{i-}=f\left(x_{k-1}^{i}, u_{k-1}\right) \quad k \geq 1, i=0, \cdots, 2 n \\
& x_{k-1}^{0}=x_{k-1} \\
& x_{k-1}^{i}=x_{k-1}+\left(\sqrt{(n+\kappa) P_{k-1}}\right)_{i} i=1, \cdots, n \\
& x_{k-1}^{i+n}=x_{k-1}-\left(\sqrt{(n+\kappa) P_{k-1}}\right)_{i}, i=1, \cdots, n \\
& x_{k}^{-}=\sum_{i=0}^{2 n} W_{i} x_{k}^{i-} \\
& P_{k}^{-}=\sum_{i=0}^{2 n} W_{i}\left(x_{k}^{i-}-x_{k}^{-}\right)\left(x_{k}^{i-}-x_{k}^{-}\right)^{T}+Q_{d}
\end{aligned}
$$

\section{UKF Correction}

$$
\begin{aligned}
& x_{k}=x_{k}^{-}+K_{k} \tilde{y}_{k} \\
& K_{k}=P_{k}^{-} H_{k}^{T}\left(H_{k} P_{k}^{-} H_{k}^{T}+R_{d}\right)^{-1} \\
& H_{k}=\sum_{i=0}^{2 n} W_{i} \tilde{y}_{k} \tilde{y}_{k}^{T} \\
& \tilde{y}_{k}=z_{k}-z_{k}^{-} \quad k \geq 1 \\
& z_{k}^{-}=\sum_{i=0}^{2 n} W_{i} z_{k}^{i-} \\
& z_{k}^{i-}=h\left(x_{k}^{i-}, u_{k}\right) k \geq 1, i=0, \cdots, 2 n \\
& P_{k}=\left(I-K_{k} H_{k}\right) P_{k}^{-}
\end{aligned}
$$

where $x_{k}^{i-}$ and $W_{i}$ are $2 n+1$ sigma points and their corresponding weights. $\kappa$ is a scaling parameter that controls the positions of the sigma points. 


\subsubsection{EnKF}

The EnKF uses samples (also known as ensembles) to represent and propagate the probability distributions of the states. By using a large number of samples, the probability density can be approximated with high accuracy. The EnKF can be summarized by (18) and (19) (Evensen 1994).

\section{EnKF Prediction}

$$
\begin{aligned}
& x_{k}^{i-}=f\left(x_{k-1}^{i}, u_{k-1}\right)+w_{k-1}^{i} \quad k \geq 1, i=1, \cdots, n_{\text {enKF }} \\
& z_{k}^{i-}=h\left(x_{k}^{i-}, u_{k}\right) \quad k \geq 1, i=1, \cdots, n_{\text {enKF }} \\
& x_{k}^{-}=\frac{1}{n_{\text {enKF }}} \sum_{i=1}^{n_{\text {enKF }}} x_{k}^{i-} \\
& z_{k}^{-}=\frac{1}{n_{\text {enKF }}} \sum_{i=1}^{n_{\text {enKF }}} z_{k}^{i-}
\end{aligned}
$$

\section{EnKF Correction}

$$
\begin{aligned}
& x_{k}^{i}=x_{k}^{i-}+K_{k}\left(z_{k}^{i}-z_{k}^{i-}\right) \\
& K_{k}=P_{k}^{-} H_{k}^{T}\left(H_{k} P_{k}^{-} H_{k}^{T}+R_{d}\right)^{-1} \\
& P_{k}^{-} H_{k}^{T}=\frac{1}{n_{\text {enKF }}} \sum_{i=1}^{n_{\text {enKF }}}\left(x_{k}^{i-}-x_{k}^{-}\right)\left(z_{k}^{i-}-z_{k}^{-}\right)^{T} \\
& H_{k} P_{k}^{-} H_{k}^{T}=\frac{1}{n_{\text {enKF }}} \sum_{i=1}^{n_{\text {enKF }}}\left(z_{k}^{i-}-z_{k}^{-}\right)\left(z_{k}^{i-}-z_{k}^{-}\right)^{T} \\
& z_{k}^{i}=z_{k}+v_{k}^{i}
\end{aligned}
$$

where $n_{e n K F}$ is the total number of samples, which are used to represent the distribution. The variable $w_{k-1}^{i}$ is a sample generated according to the $Q_{d}$ to simulate process noise. The symbol $x_{k}^{i}$ stands for the samples of a posteriori states. Note that using (19.c) and (19.d), the covariance matrix $P_{k}$ does not need to be expressively calculated.

\subsubsection{Basic PF}

The PF can be applied to systems with Gaussian and other distributions. A basic PF approximates a probability distribution function by a set of weighted discrete samples, as shown in (20).

$$
p\left(x_{k-1} \mid u_{1: k-1}, y_{1: k-1}\right)=\sum_{i=1}^{n_{P F}} W_{k-1}^{i} \delta\left(x-x_{k-1}^{i}\right)
$$

After processing several data snapshots, a PF often suffers from a degeneracy problem (i.e., the weight of only one particle tends to 1 while weights of all other particles tend to 0 ). To reduce the degeneracy problem, a resampling step is often added to re-disperse the discrete samples by generating a new set of particles according to the discrete distribution of (20). To detect degeneracy, the effective 
sampling size $N_{\text {eff }}$ is defined by (23). In the following simulation tests, resampling is initiated when $N_{e f f}<0.1 N_{p f}$. The basic PF process is described by the following equations (Arulampalam et al. 2002).

\section{PF Prediction}

$$
x_{k}^{i-}=f\left(x_{k-1}^{i}, u_{k-1}\right)+w_{k-1}^{i}
$$

\section{PF Correction}

$$
\begin{aligned}
& \widetilde{W}_{k}^{i}=W_{k-1}^{i} \cdot p_{v_{k}}\left(z_{k} \mid h\left(x_{k}^{i-}, u_{k}\right)\right) \\
& W_{k}^{i}=\widetilde{W}_{k}^{i} \sum_{j=1}^{n_{P F}} \widetilde{W}_{k}^{j} \\
& x_{k}=\sum_{i=1}^{n_{P F}} x_{k}^{i-} W_{k}^{i}
\end{aligned}
$$

PF Resampling if degeneracy is detected using (23)

$$
N_{\text {eff }} \approx \frac{1}{\sum_{i=1}^{n_{P F}}\left(W_{k}^{i}\right)^{2}}
$$

where $\tilde{W}_{k}^{i-}$ is prior weights of the $i^{\text {th }}$ state sample. $p_{v_{k}}\left(z_{k} \mid h\left(x_{k}^{i-}, u_{k}\right)\right)$ is the likelihood of $z_{k}$ given the prior states $x_{k}^{i-}$ and inputs $u_{k}$. The likelihood function is determined by the measurement noise model (i.e., $R_{d}$ ). Symbol $n_{P F}$ is the total number of samples that are used to represent the probability distribution of a state. 



\subsection{Preliminary Results}

In this section, dynamic simulation is carried out to compare the performance of the EKF, UKF, EnKF, and PF for the purpose of estimating dynamic states of a power system. The Power System Toolbox (PST) (Chow and Cheung 1992) was selected to generate simulation data that mimic the responses of a real system. The two-area, four-machine test system shown in Figure 6.1 (stored as d2asbeghp.m in PST) is used to generate the system dynamic responses to a three-phase fault. The fault is applied to Bus 3 on the line between Buses 3 and 101 at 1.1 seconds. The fault is cleared at 1.15 seconds at Bus 3 and at 1.20 seconds at Bus 101. To capture the dynamics and reduce integration errors, the simulation time step is chosen to be 0.001 second. The simulation during is set to be 15 seconds.

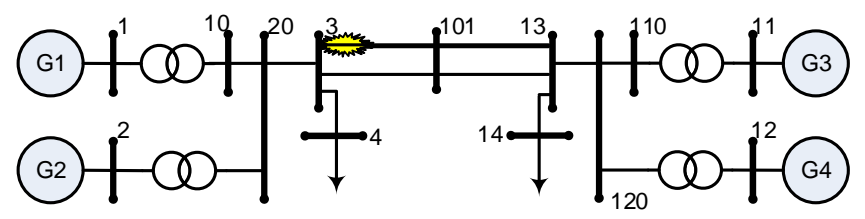

Figure 6.1. The Two-Area, Four-Machine System (Chow and Cheung1992)

Assume that PMUs are available at the generator Bus 1 to measure the voltage and the current phasors. Filtering algorithms are set up to estimate the dynamic states of Generator 1. To mimic the measurements from PMUs, the system responses are down-sampled to a rate of 25 samples per second. According to IEEE Standard C37.118-2005 (Martin et al. 2008), a certain percentage of TVEs are added to the system responses to mimic measurement noise. Because of the randomness of the measurement and process noise, the Monte Carlo methods are applied to generate $M=100$ sets of simulation data to represent various instances of random noise. The mean squared error (MSE) defined in (24) is used as a metric for comparing estimation accuracy. Here, the symbol $x_{k, \text { True }}$ represents the true state at the $k^{\text {th }}$ time step, while $\hat{x}_{k, m}$ is the corresponding estimated state in the $m^{\text {th }}$ Monte Carlo test case.

$$
\operatorname{MSE}\left(\hat{x}_{k}\right)^{\Delta}=\frac{1}{M} \sum_{m=1}^{M}\left(\hat{x}_{k, m}-x_{k, T r u e}\right)^{2}
$$

To increase the dynamic range, $10 \log _{10}(\mathrm{MSE})$ in $\mathrm{dB}$ is used to compare the algorithm performance under the following four scenarios.

\subsection{A Simple Scenario}

The goal of this scenario (Scenario A) is to set a benchmark for comparison. The simulations are set up as follows.

- To simulate the generators' dynamic responses, a fourth-order transient model as shown in (1) is used. Governors and exciters are included. Sub-transient dynamics and field saturation effects are not modeled.

- For the PMU measurements, 1.0\% measurement noise in TVE is added to the voltage and current phasors. $T_{m}$ and $E_{f d}$ are recorded with $1.0 \%$ measurement noise. The PMU sampling rate of 25 samples per second used for generating the measurement data are also used for estimation. 
- For all the algorithms, the initial states are estimated by setting $\dot{x}=0$ in (2) and then solving (2) using the Gauss-Seidel method. To reflect uncertainty of the initial states, covariance $P_{0}$ is set to be 10 times of the largest changes of the states, i.e., $P_{0}=\left(10 \max \left\{\operatorname{abs}\left[\operatorname{diff}\left(x_{1: N}\right)\right]\right\}\right)^{2}$. The output variance $R_{d}$ is set to be $0.01^{2}$ corresponding to the $1.0 \%$ of errors added, which is $\operatorname{diag}([0.01,0.01])^{2}$ for this study. $Q_{d}$ is set to be $1.0 \%$ of the largest changes of the states, i.e., $Q_{d}=1.0 \% \times \max \left\{\operatorname{abs}\left[\operatorname{diff}\left(\mathrm{x}_{1: \mathrm{N}}\right)\right]\right\}$, which is diag $([0.0474,0.0042,0.0289,0.0137])^{2}$ for this study.

The MSEs of the four states from EKF, UKF, EnKF, and PF are summarized in Figure 6.2. Both EnKF and PF use samples to represent state probability distribution. To evaluate the influence of the sample number on MSEs, 200 and 2000 samples were used for testing these algorithms. It can be observed from Figure 6.2 that the EnKF has the smallest MSE. Increasing the number of samples in EnKF does not significantly influence its estimation accuracy. In contrast, the MSEs of the PF noticeably decrease when the sample number is increased from 200 to 2000. UKF and PF have larger MSEs than the other methods.

Figure 6.3 shows the EnKF's estimation results for all 100 sets of Monte Carlo simulation with $n_{p f}=200$ samples. All 100 of the EnKF estimates converge to the true states within 1.5 seconds. Note that to help the illustration, the true value of Generator 4's rotor angle is used as the reference angle to generate the first plot of Figure 6.3. For the PF with $n_{p f}=2000$ samples, 80 sets out of the $100 \mathrm{PF}$ estimates converged and the other 20 sets diverged. When the sample number of the PF is reduced from 2000 to 200, the number of converged sets decreases to 47. For the UKF, only 67 sets of estimates converged. For the EKF, all 100 estimates converged, but they have larger MSEs than the EnKF.
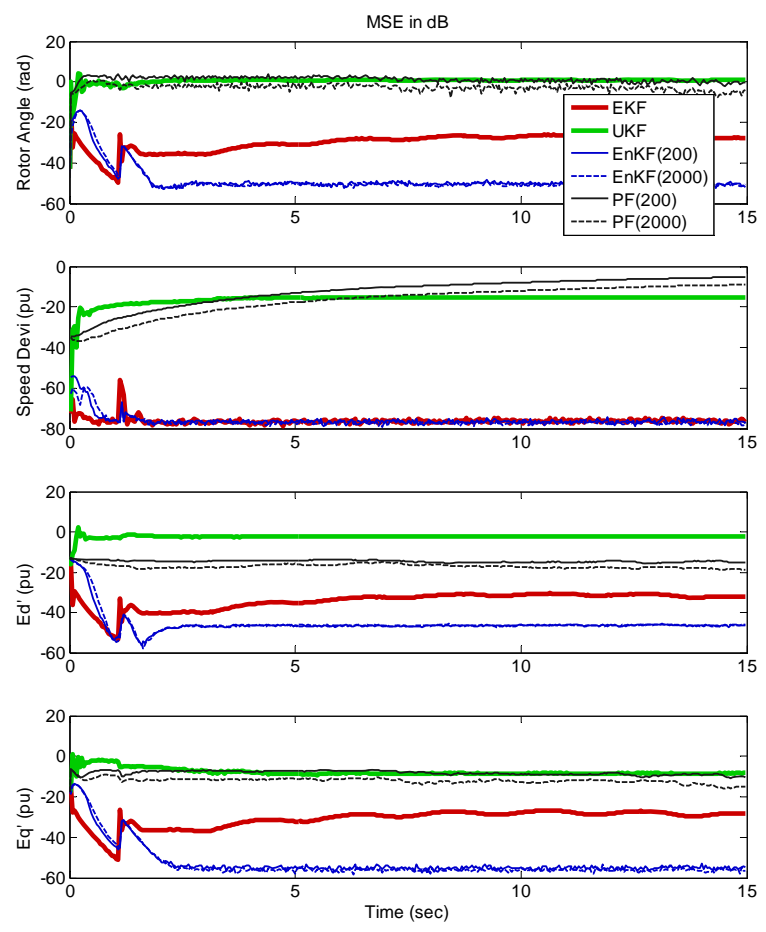

Figure 6.2. Comparison of MSEs from the EKF, EnKF, PF, and UKF for 100 Sets of Monte Carlo Simulations of a Simple Scenario 

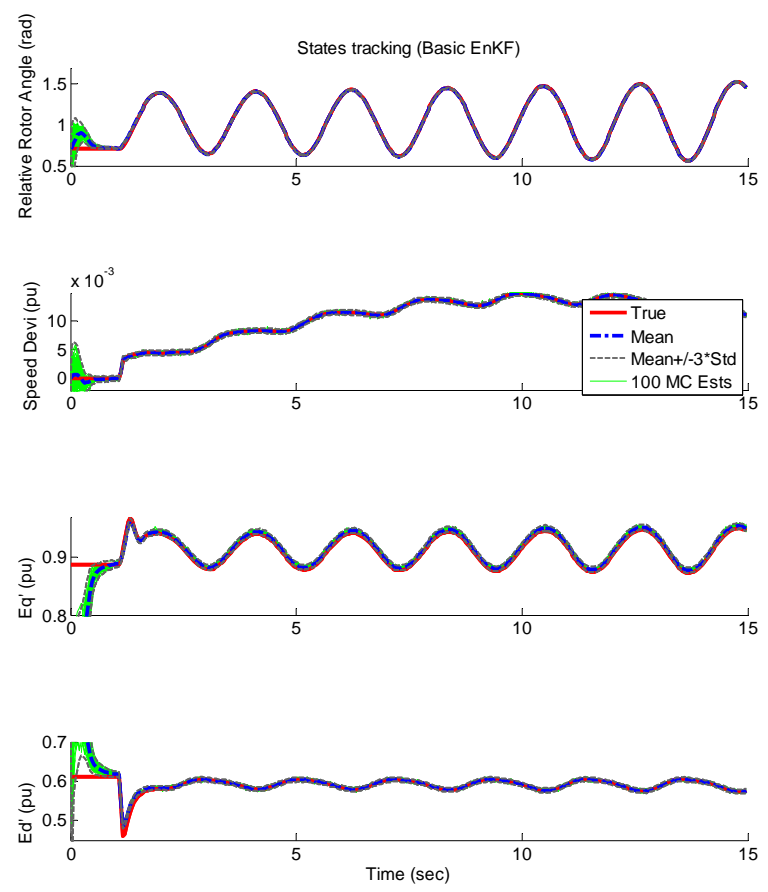

Figure 6.3. Estimated States from the EnKF with 200 Samples of 100 sets of Monte Carlo Simulations for a Simple Scenario (All 100 sets converge)

\subsection{A Scenario with Measurement Interpolation}

The goal of this scenario (Scenario B) is to evaluate how the interpolation method (Huang et al. 2003, 2009) may influence the algorithms' performance. The interpolation method inserts the additional pseudo-measurement points between two consecutive measurement samples. Introducing additional measurements increases the effective sampling rate, and reduces the linearization errors. In this scenario, the sampling rate is increased from 25 samples/s to 200 samples/s by adding seven additional pseudomeasurements between every two measurement points through linear interpolation.

Note that because of the interpolation, the sampling time interval $\Delta t$ in (7) is reduced from $40 \mathrm{~ms}$ to 5 ms. Following (10), the process noise covariance $Q_{d}$ is reduced to $1 / 8$ of that in Scenario A. The $R_{d}$ remains the same as in (12). All the rest of the setup remains the same as that in Scenario A.

The MSEs from the EKF, UKF, EnKF and PF are compared in Figure 6.4 between the cases with interpolation as in this scenario and the cases without interpolation as in Scenario A. It can be observed that the MSEs of the EKF, UKF and PF are significantly reduced with the interpolation method. In comparison, changes of MSEs for the EnKF are less significant. In addition, with interpolation, the MSEs of the EKF, UKF and EnKF are significantly smaller than those of the PF. With the interpolation method, all 100 sets of EKF, UKF, EnKF and PF estimates converge. 

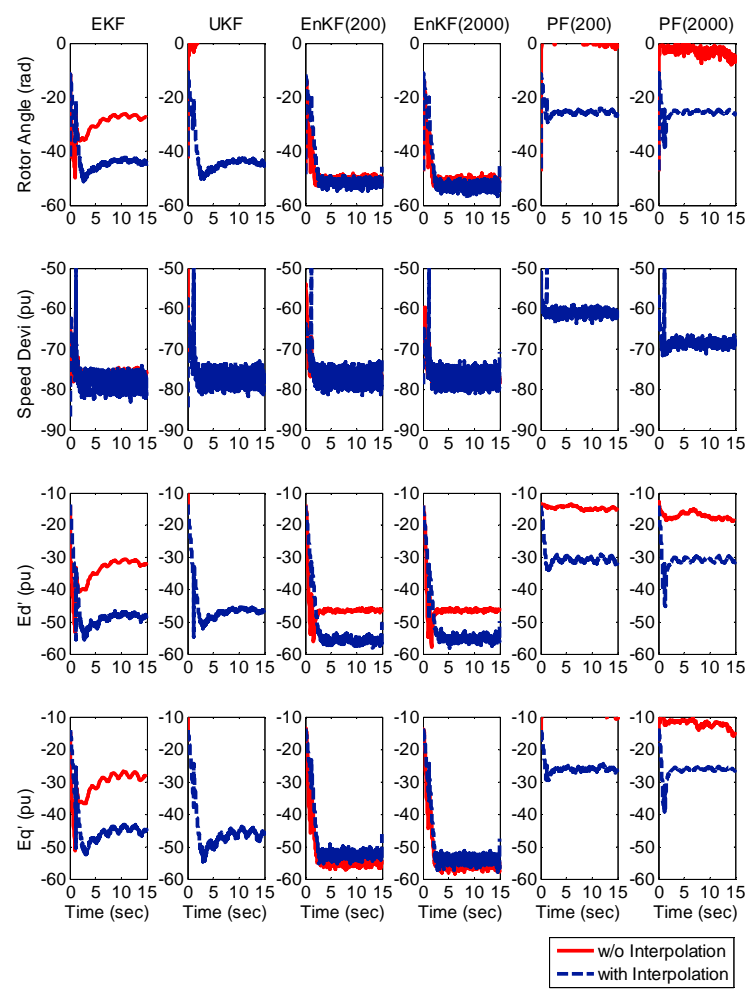

Figure 6.4. Comparison of MSEs from the EKF, UKF, EnKF, and PF of 100 sets of Monte Carlo Simulations for the Scenarios with Measurement Interpolation and without Interpolation

\subsection{A Realistic Scenario Considering Model Inadequacy}

In this scenario (Scenario C), the simulation model for generating measurement data are more complex than the estimation model used for estimating state. The goal is to mimic the more common reality where an available model is often a simplified representation of a real system. In addition, the measurement noise level is increased to include transformer noise. Finally, some model inputs (e.g., $T_{m}$ and $\left.E_{f d}\right)$ have to be estimated because they are not usually measured by PMUs.

- To simulate generator responses, a sub-transient model is used. Field saturation effects are modeled by adding $S_{1}=0.0654, S_{2}=0.4786$ (Chow 2008). To increase oscillatory dynamics, the power system stabilizers are intentionally removed. By adding the sub-transient model and saturation effects, the simulation model is more complex than the estimation model shown in (1).

- For the PMU measurements, 5\% of measurement noise in TVE is added to the voltage and current phasors. Note that additional noise is added to include measurement noise introduced by current transformers and potential transformers. In addition, because PMUs may not be available near all generators, $E_{f d}$ and $T_{m}$ are not measured in this scenario. $E_{f d}$ is estimated as a special state. $T_{m}$ is estimated by low-pass filtering $P_{e}$.

- For all the algorithms, the setup is same as that in Scenario B (e.g., the data sampling rate is increased to 200 samples/s through linear interpolation), except that the output variance $R_{d}$ is set to be $\operatorname{diag}([0.05,0.05])^{2}$ because the $5.0 \%$ measurement noise was added. 
The MSEs from the EKF, UKF, EnKF, and PF are summarized in Figure 6.5. It can be observed that the EnKF, EKF, and UKF have similar MSEs. After approximately 4 seconds, the MSEs of the PF with 2000 samples converge to similar levels. In contrast, the MSEs of the PF with 200 samples are persistently the largest among all the algorithms, indicating performance degradation. All 100 estimates from EKF, UKF and EnKF converge. All 100 estimates from PF with 2000 samples converge. In contrast, there are five sets of estimates that diverge for the PF using 200 samples.
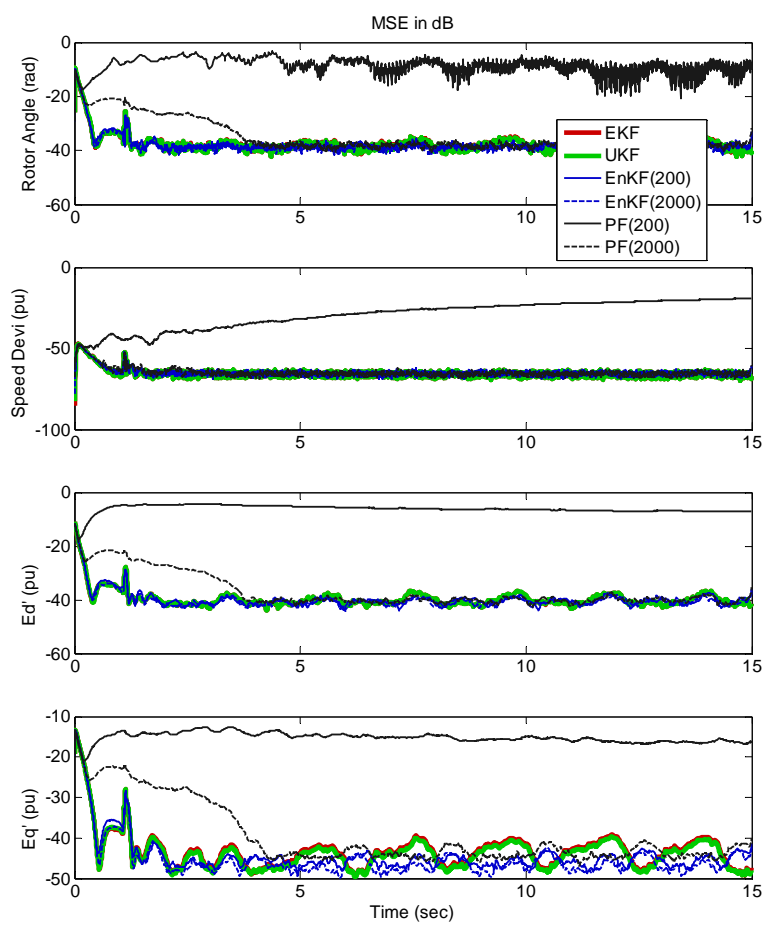

Figure 6.5. Comparison of MSEs from the EKF, UKF, EnKF, and PF of 100 Sets of Monte Carlo Simulations for the Realistic Scenario

\subsection{A Realistic Scenario with Missing Data and Outliers}

The goal of this scenario (Scenario D) is to evaluate all the algorithms when the PMU measurements are corrupted by missing data and outliers. To simulate missing data, all (simulated) measurement data between the fourth and fifth seconds is chosen to be "missing" and marked out. The missing data are then patched through linear interpolation. To mimic outliers, errors on the order of 10 times the standard deviations of the voltage magnitudes and angles are added to the voltage phasor measurements between 10.0 and 10.5 seconds. These outliers are assumed to be undetected (false negatives) and are processed as normal data by all the filtering algorithms. The remaining setup is the same as that for Scenario C.

The MSEs from the EKF, UKF, EnKF and PF are compared in Figure 6.6 between the cases with missing data and outliers as in this scenario, and the cases without missing data and outliers as in Scenario C. It can be observed that the MSEs of all the algorithms are very similar for cases with and without missing data. This indicates that all algorithms are robust against missing data that last for one second. 

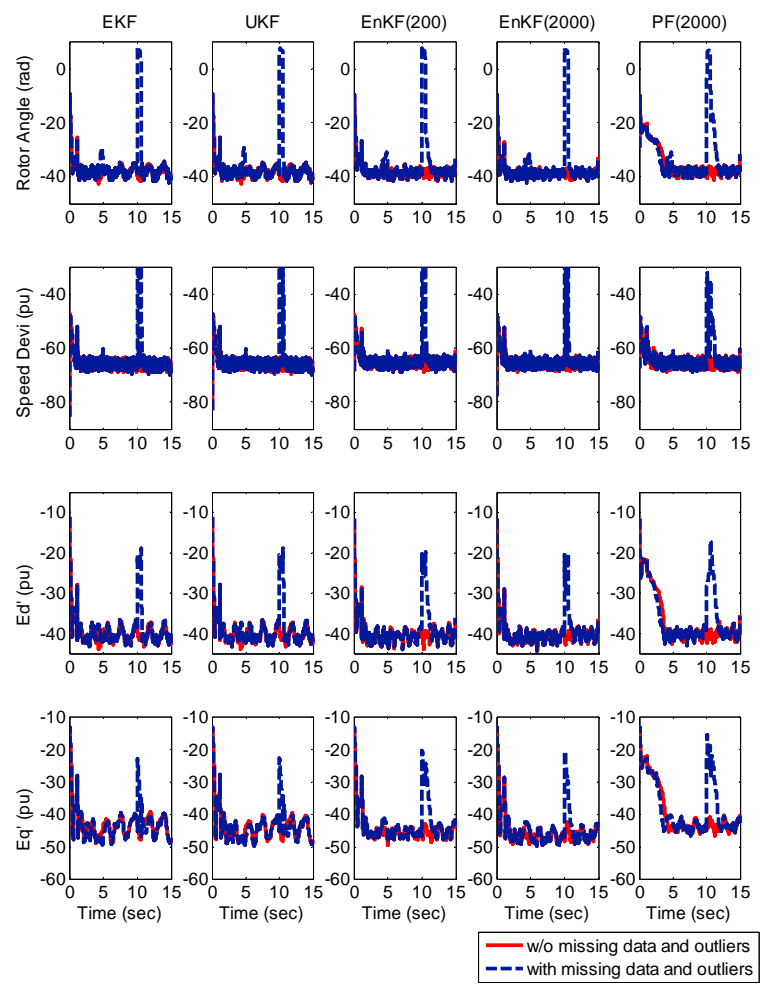

Figure 6.6. Comparison of MSEs from the EKF, UKF, EnKF, and PF of 100 Sets of Monte Carlo Simulations for the Realistic Scenario with and without Missing Data and Outliers

On the other hand, the outliers at the tenth second cause significant increases in the MSEs of all the algorithms. After the outliers disappear at 10.5 seconds, the MSEs of the EKF, UKF, and EnKF return to the original value quickly, while it takes more time for the MSEs of the PF (with 2000 samples) to return to the original values. This observation indicates that the EKF, UKF, EnKF are more robust to outliers than is the PF.

Figure 6.7 shows that all 100 sets of EnKF estimates converge to the true states. Also, the estimates of the EKF and UKF for all 100 sets of data converge. 

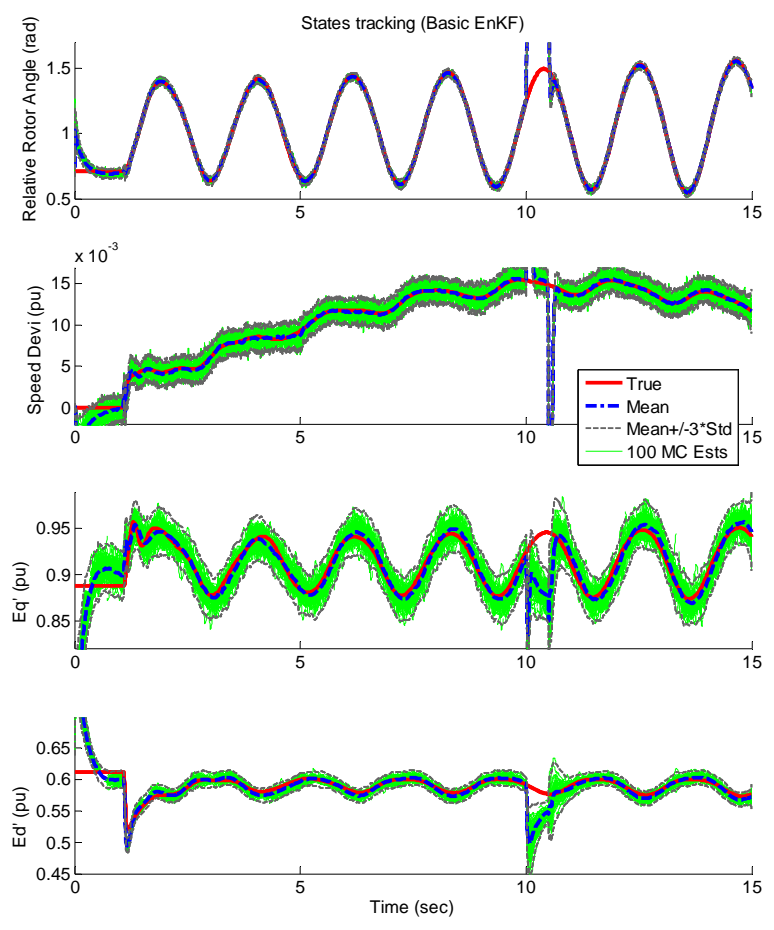

Figure 6.7. Estimated States from the EnKF of 100 Sets of Monte Carlo Simulations for the Realistic Scenario with Missing Data and Outliers (All 100 sets converge) 



\subsection{Preliminary Conclusions}

Accurate information about dynamic states is critical to efficient control of a power system, especially with the increasing complexity resulting from uncertainties and stochastic variations introduced by intermittent renewable energy sources, responsive loads, mobile consumption of plug-in vehicles, and new market designs. Using a statistical framework, this report compares the performance of an EKF, a UKF, an EnKF, and a PF for the purpose of estimating dynamic states from real-time phasor measurements. The following are shown through the simulation using a two-area, four-machine test system:

1. The EnKF algorithm outperforms other algorithms when the typical PMU sampling rate is used for estimation.

2. Measurement interpolation methods can improve the estimation accuracy of the EKF, UKF, and PF. The interpolation does not show significant influence on the performance of the EnKF.

3. Increasing the number of samples can improve the estimation and convergence of the PF.

4. All of the algorithms are robust to missing data. The outliers cause some significant errors for all algorithms if the outliers are processed as normal data. The EKF, UKF, and EnKF are more robust to the outliers than the PF. It takes more time for a PF to regain accurate state tracking after the outliers disappear than for the EKF, UKF, and EnKF. 



\subsection{Scope of Further Study and Expected Outcome}

Because of the robustness, accuracy and potential scalability of the EnKF algorithm, we choose to focus on developing EnKF algorithms while continuing to use EKF and other methods as a benchmark to compare their performance. Also, based on the preliminary study results, we propose continuing some engineering studies to explore the potentials of increasing estimation accuracy and improving execution efficiency in the areas that follow.

\subsection{Improve Algorithm Robustness against Modeling and Low Data Quality}

This study is to extend Kalman filter techniques by developing algorithms to improve estimation robustness against modeling noise and low data quality. The scope of the study is detailed as follows.

i) Modeling noise: A dynamic model is only a simplified description of a real system. Ideally, a dynamic model can approximate the corresponding system behaviors reasonably well. The difference between the model responses and real-system responses is reasonably small and can be captured by noise models. To achieve optimal performance, Kalman filters use state transition models to predict the next states and measurement models to correct the prior states. A stationary Gaussian noise model is used to describe the difference between the model and system responses. In a real-world application, it is not uncommon to encounter a large modeling noise that cannot be well described by a stationary Gaussian noise model. The planned study will improve algorithm performance by quantifying and improving modeling noise. The goal is to improve the robustness of a Kalman filter against modeling noise.

ii) Low data quality: In theory, PMUs could be deployed at every bus and provide data with minimal noise in real time at a high speed. In real-world applications, only a limited number of PMUs can be deployed. IEEE Standard C37.118-2005 (IEEE 2005) requires maximum 1\% TVE for PMU measurements. In addition, the noise from external disturbances, temporary sensor failure, temporary communication failure, current transformers and potential transformers cannot be ignored in the real-world PMU measurements. As a result, missing data, outliers, and burst noises are often observed in PMU measurements. To give an accurate state estimation, the proposed study will quantify measurement noises and improve algorithm robustness against measurement noise.

\subsection{Develop a Realistic Model for Applying and Testing EnKF using Field Measurement Data}

To gain credibility for real-world application, a dynamic state estimation method needs to be tested and evaluated using field measurement data from a real system. To achieve this goal, we will collect PMU data from a real system and establish a dynamic model corresponding to the real system. In order to fuse the data with it, a dynamic model must be established to describe a power system. PMU data from the Western Electricity Coordinating Council (WECC) are streamed into the Electricity Infrastructure Operations Center in real time and archived regularly at Pacific Northwest National Laboratory. The data are readily accessible to the team and therefore are chosen to carry out the proposed study. 
The PMU data are sparse compared to the size of the system. For example, the WECC system has more than 3,000 generators and 16,000 buses, while there are only about 522 PMUs (Silverstein 2013). With a limited number of PMUs available, it is impossible to estimate all the dynamic states for the entire system. Observability of the dynamic states can only be achieved at a limited number of devices and locations. Therefore, we plan to implement the dynamic state estimation algorithm in a local subsystem where a sufficient number of PMUs have been deployed.

In this effort, we will identify a local subsystem in the WECC system with a sufficient number of PMUs and cut it out to build a reduced-order model. In the subsystem, the voltage magnitude and angle at all buses can be measured directly or indirectly by PMUs to make sure that the subsystem has full observability. Real PMU measurements will be used with the reduced-order model for the subsystem to test the performance of EnKFs.

\subsection{Speed Up Computation and Reduce Computational Requirement with Acceptable Estimation Accuracy}

Creating a parallel version of EnKF is relatively straightforward, at least compared to the other Kalman methods, because each ensemble member can be processed independently as a series of matrix operations. The rate-limiting step is performing the Cholesky decomposition (Dereniowski and Kubale. 2004) of the quantity in parenthesis in Equation (19.b) (the Cholesky decomposition is twice as fast as an LU decomposition [Gobub and Van Loan 1996]) and scales as the cube of the number of measurements, $m$, which is twice the number of buses. If the data error covariance matrix $R$ is diagonal (the errors are uncorrelated), then the Sherman-Morrison-Woodbury formula (Woodbury 1950) can be used to further reduce the scaling so that it is only linear in the number of measurements, specifically $m N^{2}+n N^{2}+N^{3}$ instead of $m^{3}$, where $N$ is the number of ensembles (approximately the number of generators) and $n$ is the number of states, which is a small multiple (typically 2 ) of the number of generators.

The largest problem solved so far has 2,361 generators and 16,072 buses. Using 7,168 cores, the problem could be solved in 92 seconds, a speedup of 442, but over half the time was being spent in serial sections of the code. The speedup curve was nearly flat at this point, but had not yet tipped over to where performance was actually decreasing as more cores were added. The performance of the complex matrix multiplication is somewhat lower than expected. Addressing these issues should enable productive runs with over 10,000 cores. 


\subsection{References}

Arbur A and A Gómez-Expósito. 2004. Power System State Estimation. Taylor \& Francis. ISBN:0824755707.

Al-Othman AK and MR Irving. 2006. "Analysis of confidence bounds in power system state estimation with uncertainty in both measurements and parameters." Electric Power Systems Research 76(12):10111018 .

Arulampalam MS, S Maskell, N Gordon and T Clapp. 2002. "A Tutorial on Particle Filters for Online Nonlinear/non-Gaussian Bayesian Tracking.” IEEE Transactions on Signal Processing 50(2):174-188.

Chow JH and KW Cheung. 1992. "A Toolbox for Power System Dynamics and Control Engineering Education and Research.” IEEE Transactions on Power Systems 7(4):1559-1564.

Chow J and G Rogers. 2008. User Manual for Power System Toolbox, Version 3.0, 1991-2008. (Saturation data taken from the first machine of a 50 machine model in PST.)

Dereniowski, D. and M. Kubale. 2004. "Cholesky factorization of matrices in parallel and ranking of graphs." Parallel Processing and Applied Mathematics (pp. 985-992). Springer Berlin Heidelberg.

DeRusso P, R Roy, C Close and AA Desrochers. 1998. State Variables for Engineers. Second Edition, Wiley, New York.

Evensen G. 1994. "Sequential Data Assimilation with a Nonlinear Quasi-geostrophic Model using Monte-Carlo Methods to Forecast Error Statistics.” Journal of Geophysical Research 99(C5):143-162.

Fan L and Y Wehbe. 2013. "Extended Kalman Filtering based Real-time Dynamic State and Parameter Estimation using PMU Data." Electric Power Systems Research 103:168-177.

Ghahremani E and I Kamwa. 2011a. "Dynamic State Estimation in Power System by Applying the Extended Kalman Filter With Unknown Inputs to Phasor Measurements." IEEE Transactions on Power Systems 26(4) 2556-2566.

Ghahremani E and I Kamwa. 2011b. "Online State Estimation of a Synchronous Generator Using Unscented Kalman Filter From Phasor Measurements Units" IEEE Transactions on Energy Conversion 26(4)1099-1108.

Golub, G. H. and C. F. Van Loan. 1996, Matrix Computations (3rd ed.), Baltimore: Johns Hopkins, ISBN 978-0-8018-5414-9.

Guo Y, B Zhang, W Wu and H Sun. 2013. "Accuracy evaluation indexes for power system state estimation results" Power and Energy Society General Meeting (PES), 2013 IEEE, pp.1-5.

Hoteit I and D-T Pham. 2004. "An adaptively reduced-order extended Kalman filter for data assimilation in the tropical Pacific." Journal of Marine Systems 45(3-4)173-188. 
Houtekamer PL and HL Mitchell. 1998. "Data assimilation using an ensemble Kalman filter technique." Mon. Wea. Rev. 126:796-811.

Huang Y, GV Reklaitis and V Venkatasubramanian. 2003. "A Heuristic Extended Kalman Filter based Estimator for Fault Identification in a Fluid Catalytic Cracking Unit.” Industrial \& Engineering Chemistry Research 42(14):3361-3371.

Huang Z, K Schneider and J. Nieplocha. 2007. "Feasibility Studies of Applying Kalman Filter Techniques to Power System Dynamic State Estimation.” In Proceedings of IPEC2007 - the 8th International Power Engineering Conference, Singapore.

Huang Z, P Du, D Kosterev, and B Yang. 2009. “Application of extended Kalman filter techniques for dynamic model parameter calibration," IEEE Power Engineering Society General Meeting, Calgary, Canada, pp. 1-8.

Huang Z, P Du, D Kosterev and S Yang. 2013 "Generator Dynamic Model Parameter Calibration Using Phasor Measurements at the Point of Connection," IEEE Transactions on Power Systems 28(2) 19391949.

IEEE - Institute of Electrical and Electronics Engineers. 2005. C37.118-2005 - IEEE Standard for Synchrophasors for Power Systems. Institute of Electrical and Electronics Engineers. Available at "https://standards.ieee.org/findstds/standard/C37.118-2005.html".

Kalman RE. 1960. "A New Approach to Linear Filtering and Prediction Problems." Journal of Basic Engineering 82(1):35-45.

Kundur P. 1994. Power System Stability and Control. McGraw-Hill, Inc., New York.

Martin KE, D Hamai, et al. 2008. "Exploring the IEEE Standard C37.118-2005 Synchrophasors for Power Systems.” IEEE Transactions on Power Delivery 23(4):1805-1811.

Monticelli A. 2000. "Electric power system state estimation.” Proc. IEEE, 88:262-282.

Schinkel, M., W. P. M. H. Heemels, and A. Lj Juloski. 2003 "State estimation for systems with varying sampling rate." Proceedings 42nd IEEE Conference on Decision and Control, pp. 391-392.

Shih K and S Huang. 2002. "Application of a Robust Algorithm for Dynamic State Estimation of a Power System.” IEEE Transactions on Power Systems 17(1):141-147.

Silverstein A. 2013. NASPI and Synchrophasor Technology Progress. NERC OC-PC meetings, Dec. 2013.

Wan EA and R van der Merwe. 2001. "The Unscented Kalman Filter," in Kalman Filtering and Neural Networks, Simon Haykin, ed., John Wiley \& Sons, New York.

Welch G and G Bishop. 2006. An Introduction to the Kalman Filter. University of North Carolina, Department of Computer Science, TR 95-041. Available at

http://www.cs.unc.edu/ $\sim$ welch/media/pdf/kalman_intro.pdf 
Woodbury, M.A. 1950. "Inverting modified matrices," Memorandum Report 42: 106.

Zhou N, Z Huang, Y Li and G Welch. 2012. "Local sequential ensemble Kalman filter for simultaneously tracking states and parameters." North American Power Symposium (NAPS) pp. 1-6. Institute of Electrical and Electronics Engineers.

Zhou N, D Meng and S Lu. 2013. "Estimation of the Dynamic States of Synchronous Machines Using an Extended Particle Filter." IEEE Transaction on Power Systems 28(4):4152-4161. 




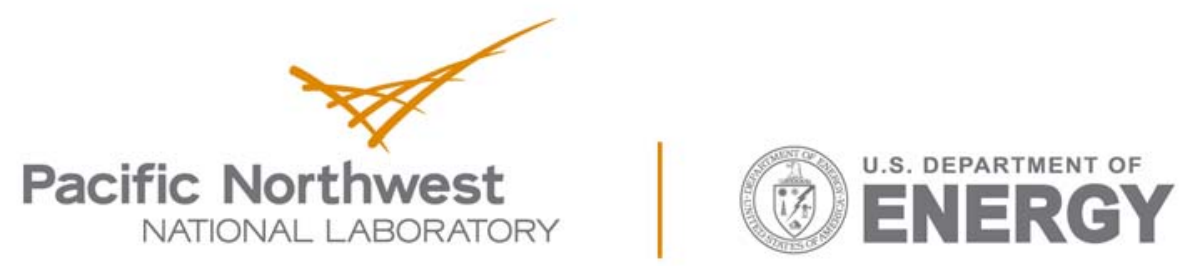

Proudly Operated by Battelle Since 1965

902 Battelle Boulevard

P.O. Box 999

Richland, WA 99352

1-888-375-PNNL (7665)

www.pnnl.gov 